\title{
Turning Hidden Nodes into Helper Nodes in IEEE 802.11 Wireless LAN Networks
}

\author{
Haithem Al-Mefleh and J. Morris Chang \\ Dept. of Electrical and Computer Engineering \\ Iowa State University, Ames, IA 50011, USA \\ \{almehai,morris\}@iastate.edu
}

\begin{abstract}
To enhance the performance of IEEE 802.11 WLANs in the presence of hidden terminal problem, we propose a protocol that allows non-hidden stations to help each other retransmit faster whenever possible. Opposite to other approaches, the new protocol benefits from the hidden terminal problem to improve the performance of DCF, which is the basic operation of IEEE 802.11. The new protocol is compatible with IEEE 802.11, and works with the same PHY of IEEE 802.11. Using Opnet simulation, results show that the proposed scheme improves throughput, delay, packet drop, retransmissions, and fairness with small trade-off regarding fairness depending on the network topology.
\end{abstract}

\section{Introduction}

The IEEE 802.11 [1,2,3] wireless networks are widely deployed. Therefore, many challenges of the wireless medium are addressed by research especially to improve the performance of the 802.11 DCF (Distributed Coordination Function), which is the basic operation of the medium access control (MAC) defined in 802.11. One major challenge is the hidden terminal problem which significantly degrades the performance of DCF because it results in high collision rates.

When a collision occurs, some stations other than the destination may be able to successfully receive one of the collided packets. Reasons include the capture effect and hidden terminal problem because of different locations of stations, existing obstacles like walls and doors, and interferences. Accordingly, and different than other proposals, we would like to investigate if non-hidden stations could help each other retransmit faster whenever possible to enhance the performance of 802.11 wireless local area networks (WLANs). In this paper, we propose a new simple protocol that modifies 802.11 DCF, is backward compatible, and works over the 802.11 PHY to achieve such goal. We also evaluate the new scheme using Opnet with and without capture effect for different topologies. Results show gains of retransmissions, throughput, fairness, delay, and packet drops with a small trade-off regarding fairness in some scenarios.

The rest of the paper is organized as following. In section 2 we provide background information about 802.11 DCF and hidden terminal problem, and then related works are discussed in section 3 In section 4 we provide details of the proposed protocol. Simulation results are given in section 5. Finally, conclusions are in section 6 . 


\section{Background}

\subsection{IEEE 802.11 DCF}

The IEEE 802.11 standard defines two mechanisms for DCF which are based on Carrier Sense Multiple Access/Collision Avoidance (CSMA/CA). In basic operation, a station that has a packet to transmit will do so if the medium is sensed idle for a period of distributed interframe space (DIFS). Otherwise, the station will go into backoff where the Binary-Exponential-Backoff (BEB) procedure is used. The station chooses a number of time slots to wait before trying to transmit again. The number, or the backoff counter, is selected from the range $[0, C W]$, where $C W$ is called the contention window and is initially set to $C W_{\text {min }}$. The station decrements its backoff counter by one for every slot time the medium is sensed idle. When the backoff counter reaches zero, the station transmits its packet. Upon receiving a data frame, the destination responds by sending back an acknowledgment (ACK) frame after a short interframe space (SIFS) time. The ACK frame has a higher priority because SIFS is the shortest interframe space (IFS) used in DCF. The packets transmitted carry the time needed to complete the transmission of a packet and its acknowledgement. This time is used by all other stations to defer their access to the medium and is called NAV, Network Allocation Vector. Collisions occur when two or more stations are transmitting at the same time, or when the ACK frame is not received after a timeout period. With every collision, the transmitting station will double its $C W$ unless it reaches a maximum limit $C W_{\max }$, and selects a new backoff counter from the new range. The process is repeated until the packet is successfully transmitted or is dropped because a retry limit is reached.

In RTS/CTS operation, a station uses control frames to contend for the channel before transmitting data frames, i.e. data frames are free of collision. When the backoff counter reaches zero, the transmitter starts by sending RTS frame to the receiver who then replies with CTS if RTS frame is received successfully. The durations of RTS and CTS frames are used to reserve the channel long enough to exchange the following data frame and its acknowledgement. Fig. 1 1illustrates the RTS/CTS operation in a fully connected (no hidden nodes) network WLAN.

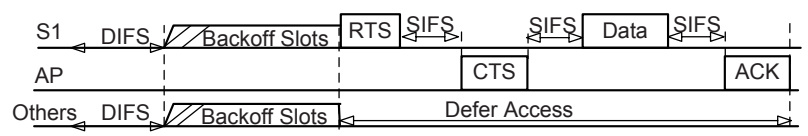

Fig. 1. RTS/CTS operation without hidden nodes

\subsection{Hidden Terminal Problem}

Using the wireless medium, a statio $\mathrm{n}$ is not able to hear frames transmitted by another station when they are out of range. Such phenomenon is referred to as the hidden terminal problem, and significantly degrades the performance of 
802.11 DCF because it results in high collision rates. An example is shown in Fig. 2 where S1 and S2 are within range, and are hidden from S3. Just like when all stations are within range, collisions occur because of equal backoff values used by different nodes. However, the hidden terminal problem adds another type of collisions as shown in Fig. 3. Here, S1 and S3 are contending for the channel with S1 backoff value is smaller than that of S3. Accordingly, S1 starts to transmit its RTS frame to the AP (access point). Unfortunately, S3 is unaware of S1's transmission and thus does not freeze its backoff counter. S1's RTS frame would not experience a collision only if S3's backoff counter reaches zero after the start of the response frame, i.e. a CTS frame from the AP. However, here S3 bacoff counter reaches zero sometime before the end of S1's transmission, and thus S3 starts transmitting its RTS frame. As a result, a collision occurs at the AP and both station S1 and S3 would timeout and then double their contention windows.
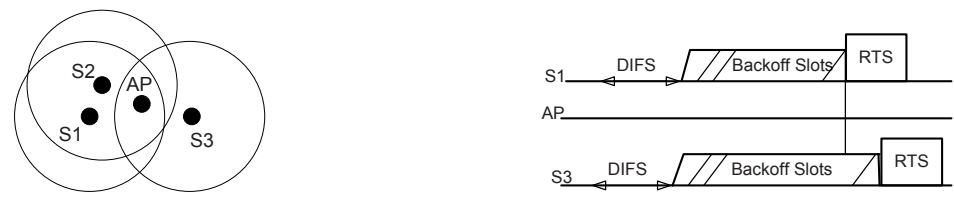

Fig. 2. Hidden terminal problem Fig. 3. Collision due to hidden terminal problem

A special situation occurs when S3 starts transmitting RTS frame at the same time the AP may start transmitting CTS frame. Accordingly, S1 would start transmitting a data frame. However, S3 would time out and begin backoff procedure. As a result, S3 may attempt to retransmit while S1 is transmitting the data frame resulting in a collision of the data frame. Consequently, data frames are not collision-free with RTS/CTS operation when hidden terminal problem exists.

\section{Related Work}

There are few analytical models for wireless networks with hidden terminals like [4, 5, 6. In 7], the authors analyze the effect of hidden terminal on the throughput of a WLAN AP. They find that hidden terminal problem reduces the network throughput by $50 \%$, and the capture effect (receiving one of the collided frames correctly under some conditions [8, 9, 10, 11, 12, ) can enhance the performance by $(10-15) \%$. Capture effect adds to the complexity and cost of wireless devices, and thus is mostly not implemented. In [13, a study of the effect of hidden terminal problem in a multi-rate 802.11 b network for both basic and RTS/CTS methods is provided. The study shows that although RTS/CTS method does not help against hidden nodes for rates higher than 1 Mbps and $2 M b p s$, it is recommended for all rates since it alleviates the packet collisions.

Different approaches are proposed to reduce the effect or/and the number of hidden nodes. First, in many protocols like 802.11 DCF [1], the RTS/CTS 
exchange is used to mitigate the hidden terminal problem. Second, the use of centralized scheduling like 802.11 PCF [1] would help. However, scheduling is not attractive because of its higher complexity, centralized control, and overhead of control packets which increase latency and reduce throughput. Third, increasing the transmission power or the carrier-sensing range may reduce the number of hidden nodes. In [12, the authors define a set of conditions for removing the hidden terminal problem for 802.11 ad-hoc and infrastructure networks: 1) the use of a sufficiently large carrier-sensing range, and 2) the use of capture effect which is referred to as the "Restart Mode" of the receiver. The authors show that one of these conditions alone is not sufficient; both conditions are required. Moreover, the work assumes that there are no significant obstructions in the network. In general, such approaches could be not desirable for energy-efficiency reasons, and would increase the exposed nodes problem in overlapping WLANs and ad-hoc networks. In addition, it may not be feasible due to different limits like available power levels, obstacles, and regulations. On the contrary, power control schemes [14,15] could result in increasing the number of hidden nodes. Fourth, multi-channel approaches [16] mitigate the effect of hidden stations. These approaches require more transceivers and channels, and more complex MAC protocols. Fifth, busy tone protocols [17,18 require a central node or a separate channel to transmit a special signal to inform other nodes that there is an ongoing data transmission. Finally, using new MACs and backoff algorithms, adapting the contention parameters, and broadcasting helpful information are used (many of which do not consider hidden-terminal problem). In [19], each station broadcasts its backoff time in data frames to achieve fairness with its neighbors, and a multiplicative increase/linear decrease backoff algorithm is used. In [20], an impatient backoff algorithm is proposed to enhance the fairness level toward the nodes in the middle of an ad-hoc network. In contrast to all existing approaches, impatient nodes decrease their backoff upon a collision or losing contention, and increase it upon a successful transmission using an exponential instead of a uniform random backoff. The authors assume slotted system where synchronization is achieved, and propose to use reset messages to address the issues of small backoff values when there are many collisions and high backoff values when there are no collisions.

\section{The Proposed Scheme}

\subsection{Motivation}

With the IEEE 802.11's distributed operation of DCF, stations compete for the channel using a random access scheme. Hence, there are always collisions whose level increases with the number of contending stations, and the existence of hidden terminal problem. Different approaches were proposed to enhance DCF by adjusting contention parameters and the backoff procedure. However as discussed in related work (Section 3), they do not eliminate the hidden terminal problem, or even do not consider it. 
When a collision occurs because of hidden terminal problem, some stations other than the destination may be able to successfully receive one of the collided packets. The same scenario may occur if the there is a bad channel between the transmitter and the destination, like existing noise at destination, while there is a good channel between the transmitter and some stations other than the destination. In the presence of hidden nodes, we would like to investigate if non-hidden stations could help each other for retransmitting collided frames to enhance the performance of infrastructure WLANs. Such cooperative retransmission is expected to be faster since with DCF a non-collided station mostly transmits earlier than collided stations that double their CW. First, we propose a new simple protocol that modifies 802.11 DCF, is backward compatible, and works over the 802.11 PHY to achieve such goal. Then, we evaluate the proposed protocol via simulation.

\subsection{Description of the New Scheme}

We distinguish between two types of transmission opportunities (TXOPs) as shown in Fig. 4. First, a normal TXOP (NTXOP) occurs when a stations starts to transmit a data frame after the required DIFS, or EIFS, and backoff periods. Second, a compensating TXOP (CTXOP) occurs when a station starts to transmit after the current NTXOP by SIFS period. Also, each station maintains locally a table, called CTABLE, of other stations that may need to be assigned CTXOPs. When a station (say S2) overhears an RTS frame or a data from another station (say S1) sent to the AP, it adds an entry (the MAC address) of the frame transmitter (S1) to its CTABLE if no such entry exists. A station (S2) drops an existing entry from local CTABLE when overhearing an ACK frame sent to another station ( $\mathrm{S} 1$ ) whose MAC address is equal to that entry. Note that a station is not required to wait for ACK frames after RTS or data frame to add/remove an entry to/from its CTABLE.

Fig. 4 illustrates the new scheme. Here, only S3 is hidden from both S1 and S2. After DIFS and backoff periods following DCF operation, both S1 and S3 transmissions overlap resulting in a collision. Since S2 overheard S1's data frame, it adds S1's MAC address to its CTABLE. After backoff, S2 transmits without interference, and at the same time informs the AP that S1 has a collided packet to transmit by including S1's MAC address in the transmitted data frame. The AP responds by sending back an ACK to S2 while piggybacking the AID of S1 in this ACK frame (CACK frame in Fig. 4). Upon receiving the ACK frame, S2 remove the entry of S1 from its CTABLE, S1 removes the entry of S2 from its CTABLE if exists, and S1 recognize that it is assigned a CTXOP. Thereafter, S1 sends a data frame after a period of SIFS to the AP who then replies with an ACK (last frame in Fig. 4). When overhearing the ACK, S2 removes S1's MAC address from its CTABLE, and all stations continue their contention for the channel.

For reasons like power saving (energy will be consumed for every bit transmitted or received), an 802.11 station first receives the MAC header of a frame and then receive the payload only if the frame is destined to that station. This be- 


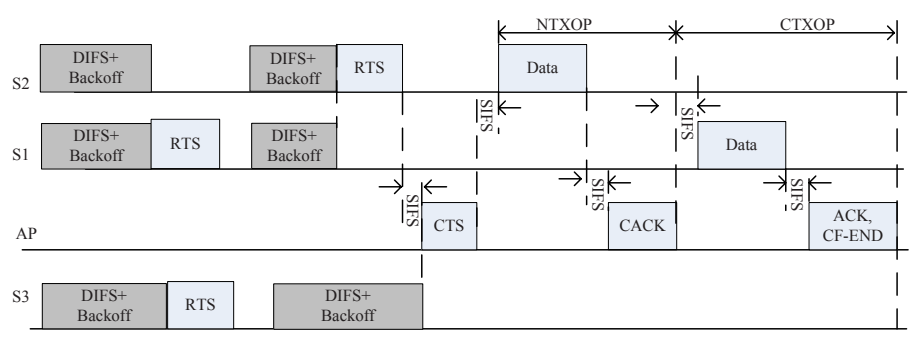

Fig. 4. The proposed scheme

havior is not changed by the new scheme as only headers information is needed. Also, the helping station does not reserve the channel for a CTXOP, but the AP does so using the duration value of the CACK frame. Since duration is not known in advance, it is set to the time required to transmit a frame with maximum possible length and lowest rate. If needed (duration reserved is longer than CTXOP), the AP sends an ACK +CF-END frame instead of ACK frame in the CTXOP so that all stations reset their NAV values to start contention. On the other hand, the AP sends a CF-END if the helped station did not start transmitting after PIFS. Finally, when a station gets a CTXOP, it does not reset its CW value and it uses its current backoff counter for the next frame in order to maintain adapting to congestion levels.

\subsection{Capture Effect}

Capture effect [8, 9, 10, 11, 12, allows receiving one of the collided frames correctly under some conditions, and thus would enhance the throughout of the network while decreasing the fairness level. Our scheme is expected to improve the performance of WLANs with or without the hidden terminal problem when capture effect is enabled since more than one station is included in a collision; those transmissions not captured still can be helped using the proposed scheme as different stations would capture different frames depending on the distance and environment between each receiver and different transmitters.

\subsection{Implementation Issues}

CACK frame is a new ACK type with a format shown in Fig. 6, and adds only one field, named CAID, to that standard ACK frame shown in Fig. 5. CAID represents the AID of the station that is assigned a CTXOP following the current NTXOP. The 16-bit AID is used because of its smaller size compared to that of the 48-bit MAC address, and thus reducing the extra time required.

To distinguish between ACK and CACK frames, we used the fact that all bits $B 8$ to $B 15$ except for $B 12$ in the Frame Control field of IEEE 802.11 control frames are always set to ' 0 '. In our implementation, we selected $B 10$ to be set 


Octets: 2
\begin{tabular}{|l|l|l|c|}
\hline $\begin{array}{l}\text { Frame } \\
\text { Control }\end{array}$ & Duration & RA & FCS \\
\hline
\end{tabular}

Fig. 5. ACK frame

Octets: 2
\begin{tabular}{|l|l|l|l|r|}
\hline $\begin{array}{l}\text { Frame } \\
\text { Control }\end{array}$ & Duration & RA & CAID & FCS \\
\hline
\end{tabular}

Fig. 6. CACK frame

to ' 1 ' for CACK. Note that a CTS frame also can be used with the same modifications to implement a CACK. The new scheme is fully backward compatible since CACK is of known type and subtype, and will not be used to acknowledge data frames from stations that do not implement the new scheme.

Data frames are not changed. AIDs cannot be used here becuase a non-AP station maintains only its own AID. Hence, the 48-bit "Address4" of the IEEE 802.11 data frame's header can be used by a station to inform the AP about a collided station.

\section{Performance Evaluation}

This section presents the simulation we used to evaluate the performance of the proposed scheme and compare it to that of 802.11 DCF. We implemented the new scheme with the commercial Opnet Modeler 11.5.A [21] by modifying the Opnet 802.11 models. We consider an infrastructure network which consists of one AP and a number of stations that share a single wireless channel. Moreover, there are no channel errors; collisions are the only source of errors. For each scenario, the results are the average of 100 different runs with a different seed, which is used for the random generator, for each run. Finally, 802.11b and RTS/CTS operation are used with the parameters shown in Table 1.

Table 1. Network Parameters

\begin{tabular}{llll}
\hline Parameter & Value & Parameter & Value \\
\hline Slot Time & $20 \mu \mathrm{s}$ & MAC ACK Size & 14 Bytes \\
SIFS & $10 \mu \mathrm{s}$ & MAC CTS Size & 14 Bytes \\
DIFS & $50 \mu \mathrm{s}$ & MAC RTS Size & 20 Bytes \\
CW $W_{\min }$ & 32 & PLCP Overhead & $192 \mu \mathrm{s}$ \\
CW & 1023 & DCF MAC Overhead 28 Bytes \\
Control Rate & 1 Mbps & Short Retry Limit & 4 \\
Data Rate & $11 M b p s$ & Long Retry Limit & 7 \\
\hline
\end{tabular}

\subsection{Performance Metrics}

For performance analysis, we use the following metrics:

1. Throughput $(S)$ : the total data bits transmitted successfully per the simulation time. 
2. Fairness Index (FI): we used Jain Index 22] defined by $\left(F I=\frac{\left(\sum_{i=1}^{n} S_{i}\right)^{2}}{n \sum_{i=1}^{n} S_{i}^{2}}\right)$, where $n$ is number of stations and $S_{i}$ is the throughput of station $i$. The closer the value of $F I$ to 1 , the better the fairness provided. We use $F I$ to find how fair a scheme is to different DCF users.

3. Average Delay: the delay of a data packet is measured from the moment it was generated until it is successfully received. Only successfully transmitted packets are considered for finding the average delay.

4. Packet drop: number of data packets dropped due to buffers overflow, and due to reaching a retry limit.

5. Retransmissions: the number of retransmission attempts of each packet before it is successfully transmitted or dropped.

\subsection{Hidden Groups without Capture Effect}

Here, each scenario is an infrastructure network with one AP and a number of stations that are positioned to be in two groups. Stations of different groups are hidden from each other, and stations of the same group are non-hidden. Each scenario is referred to as $n$ - $m$, with $n$ stations in the first group and $m$ stations in the second group, and $n$ is fixed while $m$ is variable. Then we test with scenarios referred to as $n-m-c$, where a third group (group $c$ ) of 5 stations, which are not hidden from each other, is added to each of the previous $n-m$ networks. However, stations are arranged as following: 1) $c_{1}$ and $c_{2}$ are nonhidden from all stations in network. 2) $c_{3}-\left\{n_{2}\right\}$. 3) $c_{4}-\left\{n_{1}, m_{1}, m_{5}, m_{9}, m_{10}\right\}$. 4) $c_{5}-\left\{m_{1}, m_{5}, m_{6}, m_{7}, m_{9}, m_{10}\right\}$. Here, $x_{i}$ is station $i$ in group $x$, and $x_{i}-\left\{x_{j}\right\}$ means that $x_{i}$ and $x_{j}$ are hidden from each other. Also, $|x|$ is number of stations in group $x$. These scenarios include a general topology of a wireless network. Results are provided in Fig. 7 to Fig. 14. In all figures, the letter "d" ("e") is used if the new scheme is disabled (enabled).

For the $n-m$ scenarios, different measures follow the same trend for DCF with the new scheme enabled or disabled; we show this for fairness and throughput in Fig 7 to Fig.9. This can be explained by the fact that $C W$ resetting and backoff counters are unchanged after a CTXOP. Fig. 7 to Fig. 9 also show a trade-off

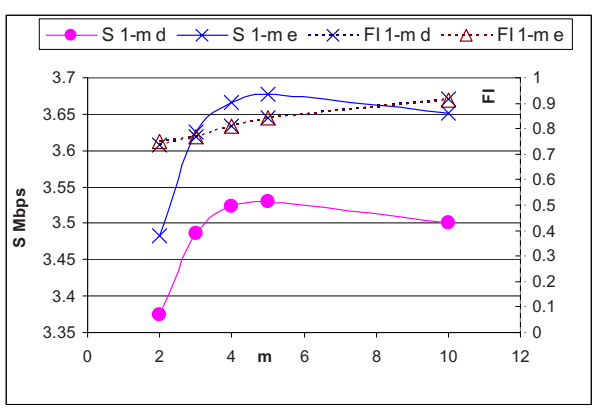

Fig. 7. 1-m

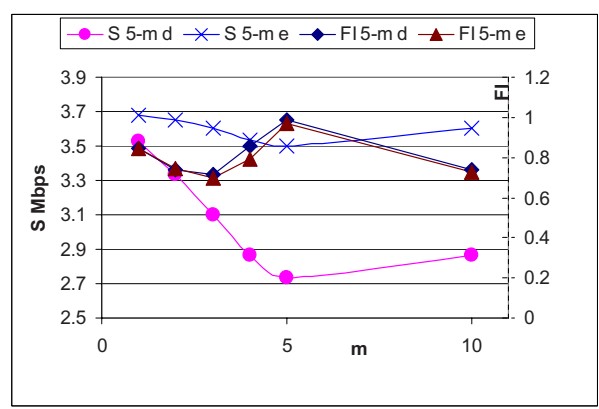

Fig. 8. 5-m 
between throughput and fairness for the $n-m$ scenarios. The fairness gets smaller for some cases when the new scheme is enabled. This is because collided stations may retransmit before being helped due to random backoff values. However, the difference is small and FI of the new scheme is always above 0.7 , and almost is the same as that of DCF for the 1- $m$, and 10- $m$ scenarios. On the other hand, fairness is always enhanced for the $n-m-c$ scenarios, Fig. 10 and Fig. 11, where there is a higher probability of being helped before retransmitting using contention due to more general relations (not just two groups). Fig. 7 to Fig. [11 illustrate that

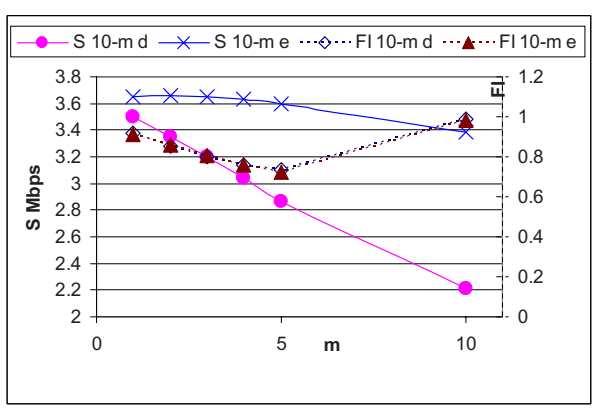

Fig. 9. 10-m

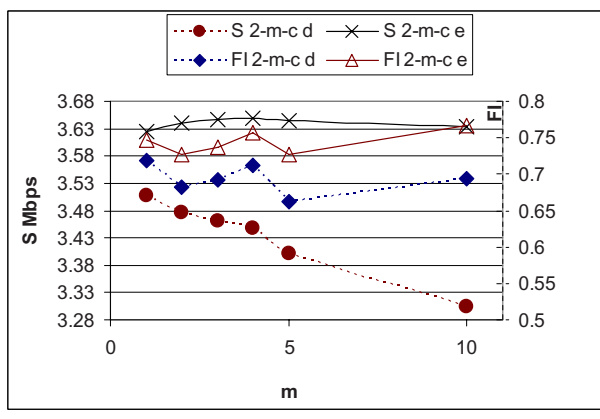

Fig. 11. 2-m-c

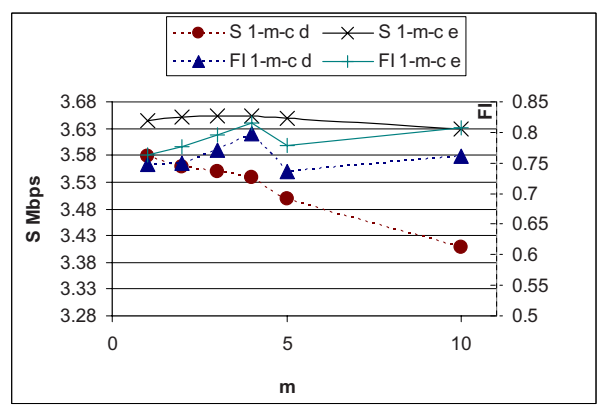

Fig. 10. 1-m-c

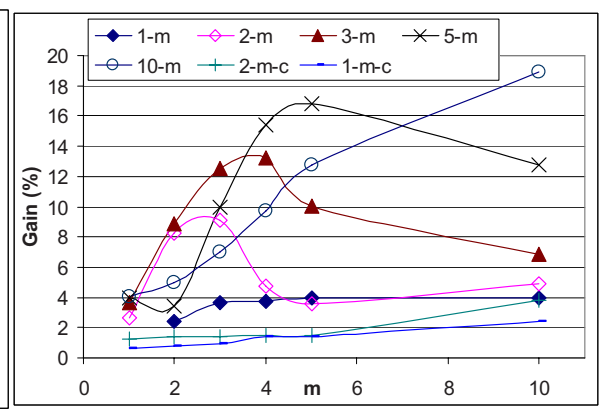

Fig. 12. Delay

throughput is always enhanced. The minimum (maximum) gains (\%) are about 3.2 (4.3), 4.1 (27.8), 4.3 (52.7), 1.8 (6.4), 3.3 (9.9) for the 1- $m, 5-m, 10-m, 1-m-c$, and $2-m-c$ scenarios respectively. Also, the throughput is always above $3.4 \mathrm{Mbps}$ when the new scheme is enabled, and may reach $2.2 \mathrm{Mbps}$ otherwise for the $n-m$ networks. In addition, throughput of $n-m-c$ networks is always above $3.6 \mathrm{Mbps}$ with the new scheme but keeps decreasing otherwise. Delay, retransmissions, and drops are also enhanced in all scenarios, Fig. 12 to Fig. 14. Gains come from the fast retransmissions as shown in Fig. 13. The performance of the new scheme is affected by number of stations in each group. For the $n-m$ networks, the gain (all measures except FI) increases until a maximum value, and then decreases until 


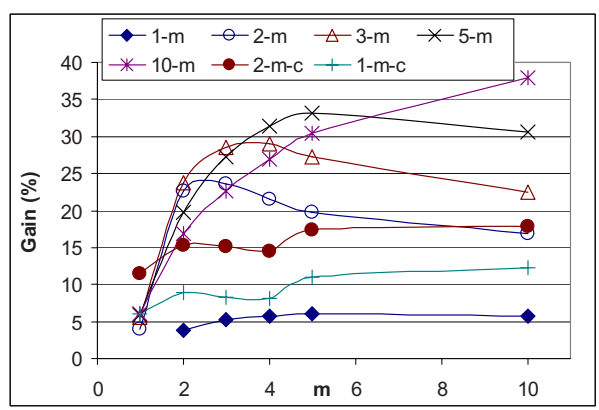

Fig. 13. Retransmissions

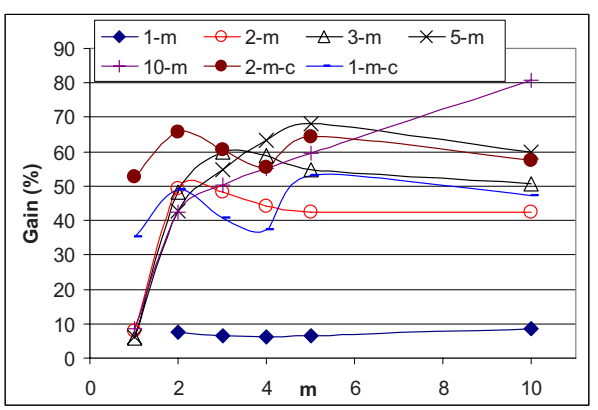

Fig. 14. Packet drop

it reaches a saturated value. This is explained by the fact that the probability of collisions due to hidden nodes decreases when $|n|$ is small compared to $|m|$ (or $|m|$ is small compared to $|n|)$.

\subsection{Random Scenario with Capture Effect}

When considering capture effect for scenarios in previous section, collected results showed similar gains but higher values of different measures in both schemes. Therefore, we do not show those results. We also randomly generated a network of 30 stations positioned around the AP which is in center of an area of $420 \mathrm{~m} \times 420 \mathrm{~m}$. In addition, a signal can be captured if received power is at least 10 times greater than the received power of any other one, and SNR requirement is met according to the model used in Opnet. Also, each station follows an ON/OFF model: each period is Exponential(0.375 seconds), traffic is generated during the ON period with Exponential( $r$ seconds), and a packet is 1024 bytes. Changing $r$ allows for testing the network with different loads.

Results are given in Fig. 15, For very small loads, there are almost zero collisions and the number of transmitters, and so helpers, is smaller. Thus no

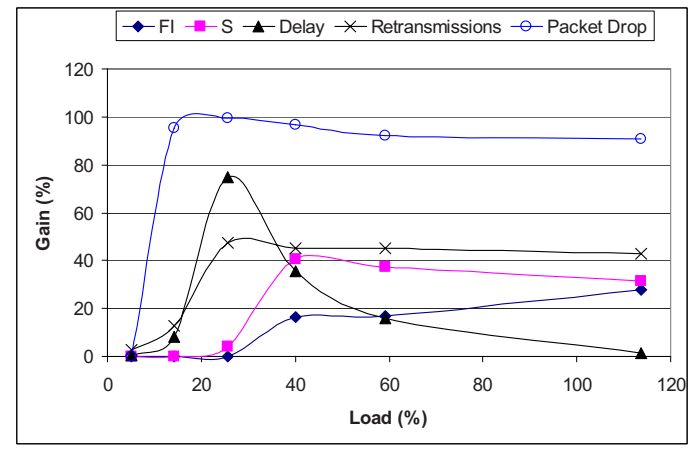

Fig. 15. Performance gain for random scenario with capture effect 
improvement is seen for such loads. However, improvements start at about loads of $14 \%$ for throughput and fairness, and at about $5.3 \%$ for all other measures. The gains (except FI which continues to increase) increase with load until a maximum value, and then start to decrease. The decrease is because when loads are higher, collisions due to hidden and non-hidden nodes also gets higher (our proposed algorithm does not change collisions), and also more packets are buffered at different stations (more delays and drops due to long waiting).

\section{Conclusions}

We proposed a new protocol for 802.11 WLANs to take advantage of the hidden terminal problem by allowing non-hidden stations to assist each other retransmit faster whenever possible. The new scheme is a modification to DCF, is backward compatible, and works over the 802.11 PHY. We evaluated the proposed scheme via simulation which was conducted using Opnet Modeler for different scenarios. Results showed that the new scheme improves the throughput, delay, packet drop, fairness, and retransmissions. The performance gain comes from cooperative retransmissions that are faster than that used in DCF where a collided station doubles its CW. In addition, results showed a trade-off between throughput and fairness only in some scenarios. Further work includes investigating performance enhancements using different design issues like having the AP decide when not to allow stations to assist each other, and using help information to update backoff counters and CW.

\section{References}

1. IEEE Std 802.11b-1999, Wireless LAN Medium Access Control (MAC) and Physical Layer (PHY) Specifications: Higher-Speed Physical Layer Extension in the 2.4 $\mathrm{GHz}$ Band (1999)

2. IEEE Std 802.11g-2003, Part 11: Wireless LAN Medium Access Control (MAC) and Physical Layer (PHY) specifications Amendment 4: Further Higher Data Rate Extension in the $2.4 \mathrm{GHz}$ Band (2003)

3. IEEE Std 802.11e-2005, Part 11: Wireless LAN Medium Access Control (MAC) and Physical Layer (PHY) specifications. Amendment 8: Medium Access Control (MAC) Quality of Service Enhancements (2005)

4. Tsertou, A., Laurenson, D.: Revisiting the hidden terminal problem in a csma/ca wireless network. In: IEEE Transactions on Mobile Computing (Accepted 2007)

5. Wu, H., Zhu, F., Zhang, Q., Niu, Z.: Analysis of ieee 802.11 def with hidden terminals. In: GLOBECOM, IEEE, Los Alamitos (2006)

6. Ray, S., Starobinski, D., Carruthers, J.B.: Performance of wireless networks with hidden nodes: a queuing-theoretic analysis. Computer Communications 28(10), 1179-1192 (2005)

7. Zahedi, A., Pahlavan, K.: Natural hidden terminal and the performance of the wireless lans. In: Proc. IEEE 6th Int. Conf. on Univ. Pers. Comm, pp. 929-933 (1997)

8. Nadeem, T., Ji, L., Agrawala, A.K., Agre, J.R.: Location enhancement to ieee 802.11 def. In: INFOCOM, pp. 651-663. IEEE, Los Alamitos (2005) 
9. Durvy, M., Dousse, O., Thiran, P.: Modeling the 802.11 protocol under different capture and sensing capabilities. In: INFOCOM, pp. 2356-2360. IEEE, Los Alamitos (2007)

10. Klienrock, L., Tobagi, F.: Packet switching in radio channels: Part i-carrier sense multiple access modes and their throughput delay characteristics. IEEE Transaction on Communication COM-23(12), 1416-1430 (1975)

11. Lee, J., Kim, W., Lee, S.-J., Jo, D., Ryu, J., Kwon, T., Choi, Y.: An experimental study on the capture effect in 802.11a networks. In: WiNTECH 2007, Montréal, Québec, Canada (September 10, 2007)

12. Jiang, L.B., Liew, S.C.: Hidden-node removal and its application in cellular wifi networks. IEEE Transactions on Vehicular Technology 56(5), Part 1, 2641-2654 (2007)

13. Borgo, M., Zanella, A., Bisaglia, P., Merlin, S.: Analysis of the hidden terminal effect in multi-rate ieee 802.11b networks (September 2004)

14. Ho, I.W.-H., Liew, S.C.: Impact of power control on performance of ieee 802.11 wireless networks. IEEE Transactions on Mobile Computing 6(11), 1245-1258 (2007)

15. Qiao, D., Choi, S., Jain, A., Shin, K.: Adaptive transmit power control in ieee 802.11a wireless lans. Proc. of the IEEE Semiannual Vehicular Technology Conference 2003 (VTC 2003-Spring) 1, 433-437 (2003)

16. So, J., Vaidya, N.H.: Multi-channel mac for ad hoc networks: handling multichannel hidden terminals using a single transceiver. In: Murai, J., Perkins, C.E., Tassiulas, L. (eds.) MobiHoc, pp. 222-233. ACM Press, New York (2004)

17. Klienrock, L., Tobagi, F.: Packet switching in radio channels: Part 11-the hidden terminal problem in carrier sense multiple access and the busy tone solution. IEEE Transactions on Communications COM-23(12), 1417-1433 (1975)

18. Haas, Z., Deng, J.: Dual busy tone multiple access (DBTMA)-a multiple access control scheme for ad hoc networks. IEEE Transactions on Communications 50(6), 975-985 (2002)

19. Bharghavan, V., Demers, A., Shenker, S., Zhang, L.: Macaw: A media access protocol for wireless lans. In: Proc. ACM SIGCOMM, London, UK (October 1994)

20. Gupta, R., Walrand, J.: Impatient backoff algorithm: Fairness in a distributed ad-hoc mac. In: ICC (2007)

21. Opnet, Opnet Modeler, http://www.opnet.org

22. Jain, D.R.: Chiu, and W. Hawe, A Quantitative Measure of Fairness and Discrimination for Resource Allocation in Shared Computer Systems, DEC Research Report TR-301 (September 1984) 